\title{
Survey on Handling Telephone Consultations at a Kampo Outpatient Clinic in Japan - Difficult Consultations and Their Handling by Outpatient Nurses
}

\author{
Jun Koike ${ }^{1, ~ *}$, Takao Namiki ${ }^{2}$, Hisashi Fujita ${ }^{3}$, Yoshiro Hirasaki ${ }^{2}$ \\ ${ }^{1}$ Department of Nursing, Faculty of Sports and Health Science, Daito Bunka University, Saitama, Japan \\ ${ }^{2}$ Department of Japanese Oriental (Kampo) Medicine, Graduate School of Medicine, Chiba University, Chiba, Japan \\ ${ }^{3}$ Department of Nursing, Niigata College of Nursing, Niigata, Japan
}

Email address:

junk@ic.daito.ac.jp (J. Koike)

${ }^{*}$ Corresponding author

\section{To cite this article:}

Jun Koike, Takao Namiki, Hisashi Fujita, Yoshiro Hirasaki. Survey on Handling Telephone Consultations at a Kampo Outpatient Clinic in Japan - Difficult Consultations and Their Handling by Outpatient Nurses. American Journal of Nursing Science.

Vol. 9, No. 4, 2020, pp. 204-210. doi: 10.11648/j.ajns.20200904.16

Received: May 4, 2020; Accepted: May 28, 2020; Published: June 4, 2020

\begin{abstract}
Aim: In order to clarify how nurses respond to telephone consultations in a Kampo medicine outpatient clinic, a fact-finding survey was conducted on the difficulties involved in handling telephone calls. Methods: The subjects were two outpatient nurses. The contents of consultations that the nurse could not handle alone were extracted from the descriptive data of the telephone calls that each nurse was not able to handle and categorized. Guidelines developed in conjunction with physicians were introduced to clarify and address the causes of the difficulty. One year later, we again administered the telephone response survey so as to compare the results with those of the first survey. Results: Telephone consultations that were difficult for nurses to handle were classified into four categories: 1) consultations about physical disorders, 2) questions about prescribed Kampo medicines, 3) reports and questions based on the patient's own judgment, and 4) questions about treatment and hospitalization. The questions about Kampo medicines related to their "continued administration" and "mix-ups of drugs," and the questions based on patients' self-judgments related to the "reduction of" or "change in" Kampo medicines. One year later, the number of such cases declined to less than half, and no telephone consultations were made within one week after the first visit. Conclusion: As a result of the creation and introduction of guidelines in consultation with physicians, outpatient nurses are now able to answer questions and receive fewer phone calls after patients' first visits.
\end{abstract}

Keywords: Fact-finding Surveys, Kampo Medicine Outpatient Clinic, Nurses, Telephone Consultations

\section{Introduction}

According to a survey on Kampo medicine conducted in recent years, $89 \%$ of physicians in Japan prescribe Kampo medicines, and there are increasing opportunities for nurses to engage with patients taking Kampo medicines [1]. It has been pointed out that nurses should have a correct understanding of Kampo medicines in order to prevent adverse events related to their administration, such as diminished effects due to incorrect perceptions regarding Kampo medicines [2,3].

In 2001, the Ministry of Education, Culture, Sports, Science and Technology stated in the "Model Core Curriculum for Medical Education" that "Kampo medicine can be outlined," and in 2017, Kampo medicine education was implemented in the faculty of medicine. The "Model Core Curriculum for Nursing Education" states that "Major Japanese Kampo medicine's effects, mechanisms, indications, adverse events, and nursing assistance can be outlined in the Basic Knowledge Required to Understand the Object of Nursing" [4]. Nursing education also requires education on the indications and pharmacological effects of Kampo medicine. According to a survey conducted at 469 nursing institutes nationwide, 17 institutions (3.6\%) have actually introduced lectures and 
exercises on Kampo medicine in their basic nursing education [5]. It has been found that, because there are few nurse training institutes that provide education on Kampo medicine, most nurses do not have a strong basic education in Kampo medicine [6]. In addition, according to a survey conducted on 96 participants in training sessions on Kampo medicine for nurses, only 2 nurses (2.4\%) had learned about Kampo medicine, while 24 nurses $(28.6 \%)$ provided guidance on Kampo medicine in clinical practice [7]. Because of the large number of Kampo medicine prescriptions given in clinical practice, nurses often have questions and experience difficulties related to Kampo medicine, and they generally need to learn more about Kampo medicine [5, 7, 8]. However, it is not clear what knowledge nurses should possess in order to appropriately deal with patients who actually take Kampo medicine.

In the outpatient clinic at the Department of Japanese Oriental (Kampo) Medicine at Chiba University Hospital, the number of telephone consultations with patients who received Kampo medicines and had concerns that could not be handled by the staff was higher than the number held with other outpatients in the medical department of the internal medicine department. Since the establishment of our clinic in 2005, nurses have provided individual consultations, but there have often been consultations on Kampo medicine that nurses did not feel equipped to hold, so it would often take a long time to respond to complaints about physical disorders or anxiety related to Kampo medicine. It was surmised that it was difficult for nurses to respond based on their personal experience and knowledge without sufficient knowledge of the characteristics of Kampo medicine and the importance of follow-up after taking them.

In December 2011, in order to ensure that the clinical department as a whole was aware of these issues faced by ambulatory nurses, a conference was held in which nurses were able to confirm the difficulty of their situation as a preliminary study. As a result, it became clear that the most difficult situation for nurses was responding to telephone consultations. In addition, the fact that the nurses had so far failed to demonstrate in concrete terms the difficulties that they experienced in providing telephone responses was also an issue that could not be resolved. Accordingly, in order to consider how nurses in charge of the outpatient clinics in our Clinical Department can respond in telephone consultations that they feel are difficult, a survey was conducted on the status of telephone counseling. We report that the nurses' response method was established based on the results of the investigation.

\section{Purposes}

1. To clarify the actual difficulties involved in telephone consultations in the Kampo medicine outpatient clinic.

2. To establish methods that enable nurses to provide telephone counseling for outpatients undergoing Kampo treatment.

\section{Methods}

\subsection{Study Design}

This is a research study carried out using the method of action research. In order to clarify the method by which nurses can respond in telephone consultations with patients undergoing medical examinations, physicians and nurses worked together on the basis of the results of the survey on telephone responses and developed a method to resolve the issues.

The research was conducted in three phases: "Survey of telephone responses;" "Preparation and introduction of guidelines;" and "Survey of telephone responses after one year" and development of the method by which nurses could respond to outpatients in telephone consultations. The survey period was February 1, 2012-April 30, 2013 (Figure 1).

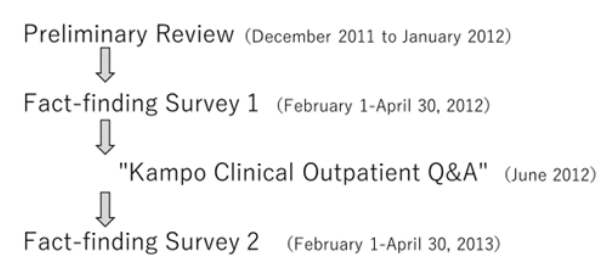

Figure 1. Progress of survey on telephone response.

\subsection{Subjects}

Two nurses in charge of outpatient clinics in the Department of Japanese Oriental (Kampo), Chiba University Hospital, agreed to cooperate in the research, and their telephone responses were recorded.

\subsection{Investigation Methods}

Two types of survey forms describing the nurses' telephone responses were independently prepared for the telephone response status survey. The form of the survey sheet was prepared by checking it using a pretest so that one telephone response could be completed within 30 seconds to 3 minutes.

At the time of the request for research cooperation, we explained to the subjects that descriptions that were as specific as possible were required for the purpose of ascertaining the actual status of the telephone responses. In consideration of the burden on the nurse based on the description in the survey form, the choices were set to reflect common telephone responses. The following two types of forms, the "Telephone response survey" and "Survey of difficult cases," were created independently and used to record results.

\subsubsection{Telephone Response Survey}

A tabular form on which 18 cases could be recorded per A4 sheet was used to record four items: (1) date, (2) time required, (3) details of inquiries from patients, and (4) answers from nurses. Two nurses recalled what was said in as much detail as possible immediately after each telephone consultation. If it took a long time to respond to the inquiry or it was difficult for one nurse to answer, only a brief note 
was left for items (3) and (4). Then, within the day of the inquiry, these details were written on the "Investigation of Difficult Case" record sheet. In addition, when checking or changing the date of an appointment or inquiring about the first visit of appointment, only choices were recorded in column (3).

\subsubsection{Survey of Difficult Cases}

In order to record responses to events that require a long period of time or are difficult for a single nurse to answer, the survey form was designed to record one case per A4 sheet using the following three items: (1) details of the inquiries made by the patient, (2) the answers given by the nurse, and (3) the patient's response. The timing of the records was not specified, and the subject was to write the records by recalling them in as much detail as possible during the day, with memos retained as necessary.

\subsection{Analytical Methods}

The number of calls handled, the time required, and the number of visits were tabulated according to the type of inquiry received from the patient.

All descriptions on the "Investigation of Difficult Cases" record sheet were transcribed, and descriptive materials for analysis were prepared. After thoroughly reading the descriptive materials, the physician categorized the descriptions based on the content because the nurse was not able to handle them alone. They were categorized according to similarities in their meanings, and the common meanings were used as category names. In the process of analyzing the descriptive contents, the adequacy of the category classifications was examined once a week by the two collaborating nurses to ensure accuracy.

\subsection{Ethical Considerations}

This study was conducted with the approval of the Ethical Review Board of the Graduate School of Medicine, Chiba University (Approval No. 1933).

The survey form used no personal patient information. All nurses in the clinical department were informed in writing and orally that the purpose, method, and personal information of the study were protected. It was explained that cooperation was voluntary, that they could participate in the study even if they declined or were unable to cooperate in the course of the study, and that they would not suffer any disadvantage if they declined.

\section{Results}

\subsection{Survey of Telephone Responses}

The number of telephone consultations held by nurses was 29 in February 2012, 40 in March, and 19 in April, totaling 88. Excluding the checks and changes of the booking dates and inquiries about the medical examination methods that the nurses responded to, there were 25 cases, of which $22(88.0 \%)$ were telephone calls that could not be handled by one nurse alone. The 22 cases were judged to be difficult cases for nurses to respond to by telephone and were categorized according to the content of the consultation. As a result, the four categories included 18 cases (24 symptoms) (Table 1), 10 cases (Table 2), 5 cases (reports and questions based on patient's own judgment) (Table 3), and 2 cases (consultations regarding future treatment and hospitalization).

The 22 phone calls took between 2 and 15 minutes, averaging 6.41 minutes. Only 8 cases involving "consultations on physical condition" and 3 cases involving "questions on prescribed Kampo medicine" were reported, while in 11 cases $(50.0 \%)$ consultation was sought regarding physical symptoms and the prescription Kampo medicine in combination.

\subsubsection{Difficulties for Patients Consulting by Telephone}

Patients who consulted the clinic by telephone for physical disorders exhibited diverse symptoms (Table 1). Questions about the prescribed Kampo medicines were divided into two categories, namely questions about the combination of their current Kampo medicine with other medicines and other Kampo medicines and questions about the continuation of their taking Kampo medicines (Table 2). The reports and questions based on the patients' self-assessments related to dose reductions of the Kampo medicine and changes to the Kampo medicine (Table 3).

Of the 22 cases, $6(27.3 \%)$ involved telephone calls within one week after the initial visit (Table 4$)$. In 4 (66.7\%) of these telephone calls within one week after the initial examination, the patients had new symptoms and asked whether they should continue taking the Kampo medicine, as they had anxiety about the symptoms. Nine of the 22 cases $(40.9 \%)$ were not immediately contacted by the attending physician and were treated at a later date.

Table 1. Telephone consultation on physical disorders: 24 symptoms

\begin{tabular}{ll}
\hline Category & Symptoms \\
\hline Whole body & Low fever, hot flashes, poor physical condition, weight gain, poor physical condition*, Cold \\
Oral cavity & Stomatitis, breath, smell \\
Digestive tract & Nausea*, Decreased appetite, Blood stool, Failure, Diarrhea Gastric pain, Strained stomach, Bad stomach \\
& Palpitations, Shortness of breath, \\
Circulatory system & Decreased blood pressure \\
Head and neck & Headache, Dizzy \\
Extremities & Numbness in the hand, Pain in the hand \\
\hline $\mathrm{N}=22$ & \\
$*$ Multiple answers &
\end{tabular}


Table 2. Questions about prescribed Kampo medicine.

\begin{tabular}{ll}
\hline Category & Contents of questions \\
\hline \multirow{3}{*}{ Continuation of medication } & Can I continue to take medication when the side effects and symptoms described in the drug information occur? \\
& The area of the headache has changed, but is it OK to continue swallowing? \\
& If the patient's physical condition deteriorates, is it OK to continue drinking (4 events). \\
& Can I take it with a drug prescribed at another hospital? \\
& The drug changed this time, but the last drug was wasted. \\
Can I take it with a new prescription? & Combination of medication with regular medication on an as-needed basis \\
& Ingestion with antibiotics. \\
\hline
\end{tabular}

$\mathrm{N}=22$

Table 3. Self-Assessment Reports and Questions.

\begin{tabular}{ll}
\hline Category & Contents of Reports and Questions \\
\hline Reduction in the dose of Kampo & The dose was reduced to two-thirds based on self-judgment, but is this enough? \\
medicine & Dose reduction of $1 / 2$ does not improve symptoms. \\
& Reports that patients are changing drugs on their own or on their own judgment. \\
& She had a cold and stopped taking the medicine and took the Kampo medicine that had been prescribed before, \\
Shange in Kampo medicine & I want to change to the previous drug.
\end{tabular}

$\mathrm{N}=22$

Table 4. Samples of telephone consultations within 1 week after initial medical examination.

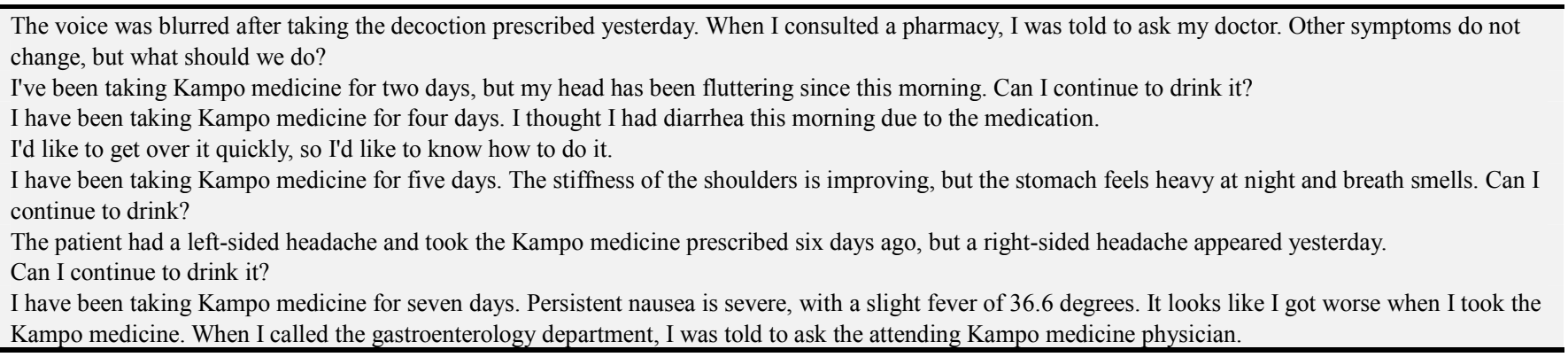
$\mathrm{N}=22$

\subsubsection{Difficulties for Nurses Responding to Telephone Counseling}

We discussed the results of the survey with the two collaborating nurses. It became clear that the patients hoped that the symptoms would improve immediately after taking the Kampo medicine, and that they thought that the effects of the Kampo medicine would be altered if symptoms of anxiety appeared. When a patient made an inquiry to a dispensing pharmacy or other department about the symptoms that he/she was worried about, he/she was advised to consult with the attending physician in the Kampo medicine department (Table 4). In addition, patients were asked by physicians in other departments to ask the attending physician in department of Kampo medicine about whether the patient could take antibiotics together with Kampo medicines. When patients consulted by telephone as outpatients at the Kampo Clinic, the nurses felt that situations in which they were unable to answer at their own discretion and unable to contact the attending physician were very challenging.

Based on the content of the consultations, it became clear that when taking Chinese herbal medicines, patients were apt to think and worry about the treatment. The nurses shared the view that it was necessary for them to understand the basics of Kampo medicine in order to respond by telephone to patients' questions or anxieties about the Kampo (Wakan) Clinic.

\subsection{Preparation and Introduction of Guidelines}

Based on the results of "Fact-Finding Survey 1," the clinical department as a whole together with the doctors at the Kampo (Wakan) Clinic concluded that it was necessary for the nurses to learn about the basics of Kampo medicine and to answer basic questions about Kampo medicine. Based on the results of "Survey 1 on Telephone Response," three physicians in the Kampo department prepared basic guidelines for how nurses should respond to inquiries from patients. These guidelines were called the "Kampo Clinic Outpatient Q\&A" (Table 5). 
Table 5. Six questions and answers from the Kampo Outpatient Clinic $Q \& A$.

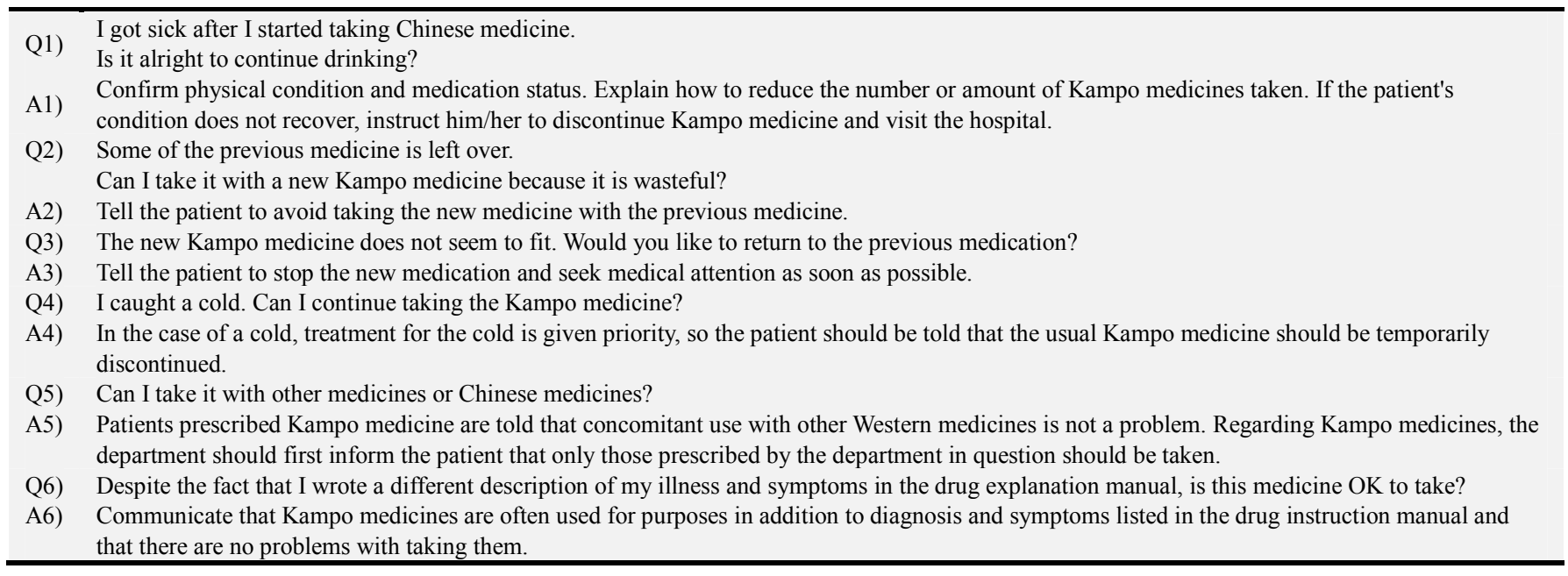

\subsection{Survey of Telephone Responses After 1 Year}

Beginning in June 2012, all of the nurses started using the "Kampo Clinic Outpatient Q\&A" at our Outpatient Clinic at Chiba University Hospital. It was used not only for telephone consultations but also for in-person outpatient consultations and questions.
The second telephone response survey conducted one year later showed there were 2 cases in February, 1 in March, and 6 in April in which the nurses found it difficult to respond. The number of cases involving difficulty in responding decreased by $59.1 \%$ from the same period in the previous year (Figure 2). The nine phone calls took 3 to 10 minutes, averaging 5.22 minutes.

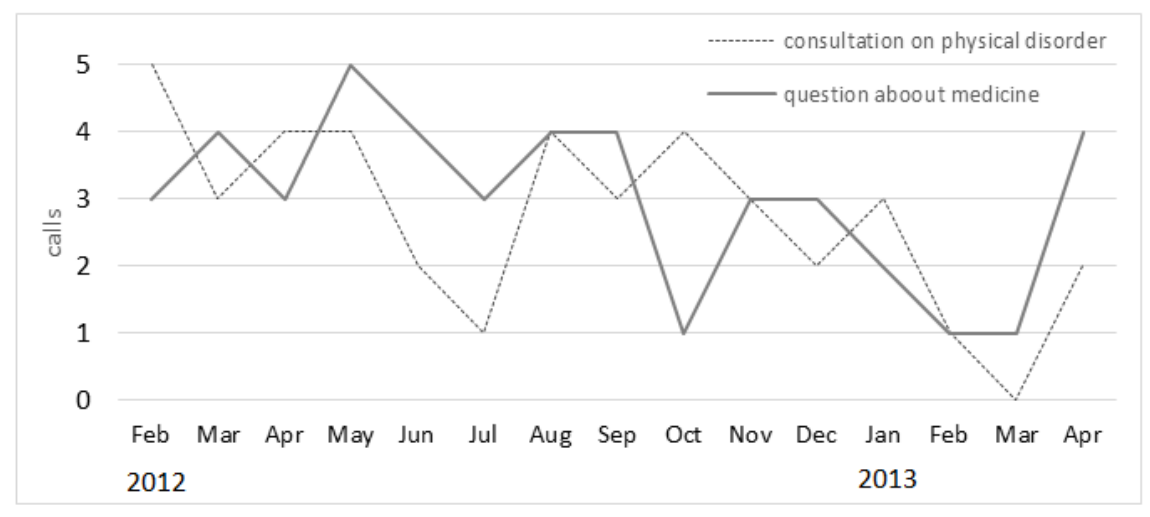

Figure 2. Number of difficult cases that nurses responded to in telephone consultations.

Of the consultations on physical disorders and Kampo medicines, the number of telephone consultations within one week after the initial consultation was 2 in February 2012, 1 in March, 3 in April, 1 in May, and 1 in August, but was 0 since September 2012. In Survey 2 conducted from February to March 2013, there were no cases of physical disorders within one week after the first visit or telephone consultations concerning Kampo medicine, representing a decrease of $100 \%$ from the same period in the previous year (Figure 3).

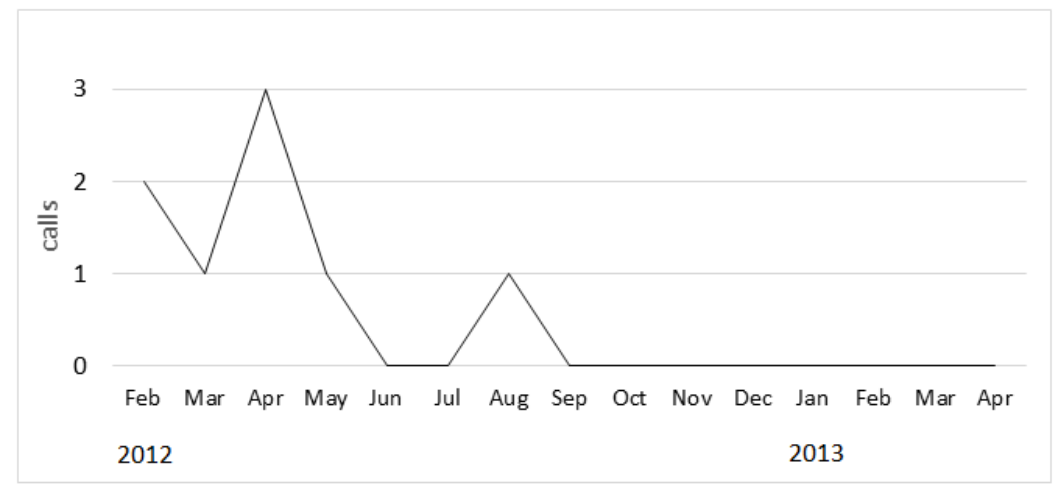

Figure 3. Number of telephone calls within one week after the initial visit. 


\section{Discussion}

The majority of patients at the Kampo medicine outpatient clinic in the Department of Japanese Oriental (Kampo) medicine at the University Hospital at Chiba University School of medicine are in their $60 \mathrm{~s}$, and their ages range from 10 years or younger to 90 years. There are approximately 2.5 times as many females as males. The diagnosed Western medical diseases (ICD-10) are characterized by musculoskeletal and connective tissue diseases, nervous system disorders, and digestive system disorders, which are particularly common. Patients suffer from multiple and complex symptoms, and Kampo medicine is often used in combination with Western medicine [9]. The results of this survey were reconfirmed. The results of this survey were not limited to the Kampo medicine clinic and the difficulty of responding to patients with these diverse conditions by telephone [10]. The difficulty of dealing with patients who had never been involved in faces and with work interruptions due to telephone calls were also reported as factors [11]. Similar factors were also observed in the present study. Based on the telephone consultation content and the nurses' feelings of difficulty, it became clear that both the patients' difficulties with continuing Kampo medicine and the inability of the nurses who first consulted by telephone to deal with the problem were important factors.

According to the results of the survey, it became clear that the most common anxiety that was caused by the complex physical symptoms that appeared after the outpatient consultation was that the nurse had previously conveyed the questions to the attending physician who had answered them. The physical symptoms that appeared after outpatient visits varied, and it was difficult to judge minor changes in symptoms or the appearance of symptoms, although in-person consultations were recommended for obvious acute symptoms. The nurses felt most uncomfortable when they could not answer a question themselves, and when they were unable to contact the attending physician immediately.

It was possible that often the onset and exacerbation of symptoms was due to the fact that the patient had started taking Kampo medicine or changed it, and the patient then became anxious and made the telephone call. Questions about the prescribed Kampo medicines were often about whether the patient should continue taking the medication due to changes in their physical condition or matching with prescription drugs for other medical departments, and it became clear that patients who took Kampo medicines were prone to anxiety and doubt. With outpatient treatment, the physician explained the prescription medication at the time of the examination, but the content of the telephone consultation showed that there were patients who returned home without having understood it at the time of the examination. In addition, when a patient asked the dispensing pharmacy about the side effects of Kampo medicine or called another department with concerns about the worsening of symptoms, they were instructed to consult with the attending physician in the Kampo medicine department, but in many cases they were not able to consult with the attending physician in the Kampo medicine department. It is also believed that the fact that Kampo medicine was promoted to the Kampo Clinic resulted in a large number of consultations by telephone. Also, the Chinese herbal medicine department called patients who were continuing treatment to confirm the appropriateness of the newly prescribed Kampo medicine after the dose was reduced, changed, or discontinued.

Thus, if a nurse has a basic grasp of Kampo medicine and shares the information with the doctor, that nurse can handle the consultation with the patient. In particular, because of the large number of consultations that occurred several days after the first visit, it was clear that the patients who had started Kampo treatment were in a difficult situation. As the need for support from nurses became clear, a guideline titled "Kampo Clinic Outpatient Q\&A" was prepared in consultation with the physicians in the clinical department to present basic knowledge on Kampo medicine in an easy-to-understand manner in order to resolve the difficulties of both the patients and nurses attending the clinic. Thanks to the guideline, nurses were able to understand patients' questions during the preparation process, acquire basic knowledge about and the rationale for Kampo medicine, and model how patients could deal with their concerns. As a result, it has become possible for nurses to respond to many questions from patients. One reason for the marked decrease in the number of telephone consultations in the survey conducted one year later is thought to be that the outpatient nurses were better able to meet the patients' needs for information and support. It was suggested that collaborative support from nurses would help patients to understand the prescribed Kampo medicine correctly and to be able to continue the treatment without anxiety.

In addition, many of the patients attending the outpatient clinic for Kampo medicines also attend other clinical departments, and many of the Kampo medicines are co-administered with Western medicines [12]. Therefore, compliance issues should be considered when patients take multiple drugs, and it is not easy for pharmacists to explain the efficacy of Kampo medicines or the purpose of prescribing them to patients in an easy-to-understand manner. To resolve this issue, databases have been established to standardize drug administration guidance [13]. If a multidisciplinary team can cooperate to support the continuation of treatment to relieve patients' anxiety and doubts about Kampo medicine, it will have a therapeutic effect.

By clarifying the difficulties specific to the department of Kampo medicine and examining methods of coping with these difficulties, outpatient nurses' attitudes have been changed, making it possible for them to comfortably respond to questions from patients. A practical and comprehensive study has shown that nurses' knowledge and awareness of Kampo medicine is changing [14]. Therefore, the content of nurses' education as it relates to Kampo medicine will be considered in the future as well. Clarifying issues in clinical 
practice will lead to the establishment of practical nursing care that can respond to patients' anxiety and doubts about Kampo medicine.

\section{Conclusion}

1. The telephone consultation for the patient was a combination of the symptoms of the patient's physical condition and the prescription Kampo Medicine. The content of the consultation was divided into questions concerning the continuation of the administration of Kampo Medicine and the combination of drugs, and reports of dose reductions and changes.

2. In the outpatient clinic for Kampo medicine, it became clear that both the difficulty of receiving prescription drugs was easier for the patient, and the difficulty was easy for the nurse who first received the consultation by telephone to deal with the problem.

3. Since patients at the start of Kampo treatment are more susceptible to anxiety due to changes in symptoms, it is necessary to improve explanations at the time of consultation.

4. The development and utilization of guidelines enabled nurses to respond to predictable questions and reduced the number of telephone consultations.

\section{Acknowledgements}

We would like to express our sincere gratitude to Nurse Michiko Sanematsu, Nurse Yuka Muramatsu, and the physicians in the Department of Kampo Medicine, Chiba University Hospital, for their cooperation in this study.

\section{Conflict of Interest}

All the authors do not have any possible conflicts of interest.

\section{References}

[1] Japanese Society of Crude Drug Preparations. Kampo Pharmaceutical Prescription Survey 2011. Available from: https://www.nikkankyo.org/serv/serv1.htm [cited 2 August 2019]

[2] Akase T. Pharmacology education in nursing. Folia Pharmacol. Jan. 2018; 151 (5): 191-194.

[3] Namiki T, Kasahara Y, Sekiya N, et al. The revealing of problems concerning about treatment of kampo formulae in hospitalization; by the analysis of questionnaires for pharmacists and nurses at ward. Kampo Med. 2009; 60 (2): 185-193.

[4] Medical Education Division, Higher Education Bureau, Ministry of Education, Culture, Sports, Science and Technology. Nursing Education Model Core Curriculum Academic Objectives Aiming to Acquire Core Nursing Practice Capacity in Bachelor's Courses. October 31, 2017. [cited 12 August 2019] Available from: http://www.mext.go.jp/b_menu/sh ingi/chousa/koutou/078/gaiyou/1397885.htm

[5] Nakano E, Yasukata F, Yamazumi Y, et al. The actual situation of the Chinese medicine medicare education in the nursing basic education. Fukuoka Prefectural University Journal of Nursing Research 2013; 10 (2): 65-71.

[6] Yakubo M, Kinoshita Y, Ueda Y, et al. Kampo medical education for university students of health education. Kampo Med. 2011; 62 (1): 65-69.

[7] Eguchi Y, Takemori S, Yoshida C, Yamada M. Recognition and learning needs of Kampo medicine of nurses. Luke's Society for Nursing Research. 2016; 20 (1): 19-26.

[8] Takemori S, Eguchi Y, Yoshida C, Yamada M. A Life-long Learning Program for Kampo Medicine for Nurses. Luke's Society for Nursing Research, 2016; 19 (2): 54-60.

[9] Takano S, Nakamura M, Morita A, et al. Usefulness of Kampo medicine for avoiding polypharmacy among hospitalized patients. Kampo Medicine. 2018; 69 (4): 328-335.

[10] Okuura K, Nakaoka K, Masui M. The relationship between the content of the telephone consultation in the outpatient department and the stress of the nurse. Japanese Society of Nursing Therapies. 2019; 49: 130-133.

[11] Hori Y, Noma Y, Kagine H, et al. Efforts to provide telephone counseling to outpatients with cancer and their families, and the current situation and challenges for establishing a patient support system. Journal of North Medical Center of Kyoto Prefectural University of Medicine 2016; 2 (1): 102-107.

[12] Ikarashi N, Shimura A, Takezawa T, et al. Survey of the use Kampo Medicines at Kampo Clinic 1 - Combined use with Western medicines -. Pharmaceutical medicine 2007; 33 (4): 353-358.

[13] Takagi A, Yoshida N, Watanabe Y, et al. Kampo Medicine Database improve Drug Information Service and Pharmaceutical Care for Inpatients in Department of Japanese oriental Medicine. Pharmaceutical medicine 2007; 33 (1): $15-22$.

[14] Kang M, Miyazono M, Hashiguchi N, et al. Factors related to health promotion behavior change of clinical nurses who took Kampo Medicine Seminar. International Nursing Care Research 2018; 17 (4): 31-40. 